\title{
Evaluation of insulin sensitivity from a minimal model adapted to oral glucose tests
}

\author{
F. Di Nardo ${ }^{1}$, F. Casagrande ${ }^{1}$, F. Carle ${ }^{2}$, M. Boemi ${ }^{3}$, P. Fumelli ${ }^{3}$, \\ P. Morosini ${ }^{4} \&$ R. Burattini ${ }^{1}$ \\ ${ }^{I}$ Department of Electromagnetics \& Bioengineering, \\ Polytechnic University of Marche, Ancona, Italy \\ ${ }^{2}$ Epidemiology Biostatistics and Medical Information Technology Centre, \\ Polytechnic University of Marche, Ancona, Italy \\ ${ }^{3}$ Metabolic Disease and Diabetes Unit, INRCA-IRCCS, Ancona, Italy \\ ${ }^{4}$ Unit of Internal Medicine, "C. G. Mazzoni” General Hospital, \\ Ascoli Piceno, Italy
}

\begin{abstract}
Recently, we proposed a new methodology for clinical evaluation of insulin sensitivity, $S_{I}$, and the rate constant of glucose absorption, $k_{a b s}$, from oral glucose tests. This methodology relies on a minimal model of glucose kinetics coupled with a structural description of gastric emptying of glucose and its conversion into the rate of systemic absorption from the gut. The aim of the present study was to test the reproducibility of $S_{I}$ and $k_{a b s}$ provided by our model using two data sets from a 300 min frequently sampled oral glucose tolerance test: a full 22-sample data set $\left(\mathrm{OGTT}_{300 / 22}\right)$ and a reduced 11-sample data subset $\left(\right.$ OGTT $\left._{300 / 11}\right)$. Nine subjects, not affected by the metabolic syndrome, participated in the study. Mean (95\% confidence interval) values of $S_{I}^{22}$ and $k_{a b s}^{22}$ obtained from the $\mathrm{OGTT}_{300 / 22}$ were $12.3(8.8-15.8) \times 10^{-4} \mathrm{~min}^{-1} /(\mu \mathrm{U} / \mathrm{ml})$ and $1.80(1.31-2.29) \times 10^{-2} \mathrm{~min}^{-1}$, respectively. Mean values of $S_{I}^{11}$ and $k_{a b s}^{11}$ obtained from the $\mathrm{OGTT}_{300 / 11}$ were $11.3(7.6-15.0) \times 10^{-4} \mathrm{~min}^{-1} /(\mu \mathrm{U} / \mathrm{ml})$ and $1.75(1.19$ 2.31) $\times 10^{-2} \mathrm{~min}^{-1}$, respectively. The concordance of the full and reduced paired samples in providing $S_{I}$ and $k_{a b s}$ estimates is supported by intraclass correlation coefficients of 0.93 and 0.97 , respectively. Parameter estimates and model based simulations of the rate of glucose appearance into plasma, $R_{a}(t)$, were comparable with data in the literature. In conclusion, our model appears a potentially useful tool to estimate insulin sensitivity and the rate constant of systemic glucose absorption from reduced oral glucose tests.

Keywords: glucose kinetics, gastric emptying, gut model, OGTT.
\end{abstract}




\section{Introduction}

The measure of an insulin sensitivity index, defined as the ability of insulin to control glucose production and utilisation is of primary importance in the assessment of glucose regulatory system efficiency. Quantitative evaluation of this index is usually accomplished with methods involving an intravenous administration of glucose and/or insulin, such as the glucose clamp or the frequently sampled intravenous glucose tolerance tests (IVGTT) and minimal model technique [1-4]. Difficulty in the venous administration and high (nonphysiological) levels of glycaemia and insulinemia achieved during these tests are limitations that need to be resolved. Measurement of insulin sensitivity from an oral test, e.g., a meal glucose tolerance test (MGTT) or an oral glucose tolerance test (OGTT), is better suited to normal life condition [5-9]. Models for interpretation of data from oral tests, however, enhance the problem of describing the rate of appearance into plasma, $R_{a}(t)$, of glucose absorbed from the gastrointestinal tract [5-9]. Recently, to estimate insulin sensitivity from an OGTT we [9] coupled the classical minimal model of glucose kinetics with a model of the gut, which describes the conversion of ingested glucose into $R_{a}(t)$. This description involves a parameter, $k_{a b s}$, which portrays the physiological meaning of a rate constant of glucose absorption by the systemic circulation from the gut. The aim of the present study was to test the reproducibility of $S_{I}$ and $k_{a b s}$ provided by our model using two data sets from a $300 \mathrm{~min}$ frequently sampled oral glucose tolerance test: a full 22-sample data set $\left(\mathrm{OGTT}_{300 / 22}\right)$ and a reduced 11-sample data subset $\left(\right.$ OGTT $\left._{300 / 11}\right)$ more suitable for population studies.

\section{Methods}

\subsection{Database}

This study included nine subjects ( 3 men and 6 women with mean age of 49.1 $(\mathrm{SD}=13.7) \mathrm{yr}$ and body mass index, $\mathrm{BMI}$, of $24.4(\mathrm{SD}=2.9) \mathrm{kg} / \mathrm{m}^{2}$, recruited at the Metabolic Disease and Diabetes Unit of the INRCA-IRCCS, Ancona, Italy, and at the Unit of Internal Medicine, "C.G. Mazzoni" General Hospital, Ascoli Piceno, Italy. These subjects were not affected by the metabolic syndrome, MS, defined according to the Adult Treatment Panel III criteria [10], and had a fasting glycaemia $<110 \mathrm{mg} / \mathrm{dl}$.

Each subject underwent an oral glucose tolerance test (OGTT). All they gave informed consent to the procedures approved by the Ethics Committee. Starting time was 8:30 a.m. after overnight fast. A $75 \mathrm{~g}$ glucose load was administered and 22 blood samples were taken: one fasting blood sample was taken immediately before glucose administration and twenty-one additional samples were taken at minutes $10,20,30,45,60,75,90,105,120,135,150,165,180$, $195,210,225,240,255,270,285$ and 300, after glucose administration $[6,8]$.

Blood was promptly centrifuged and glycaemia immediately measured by the glucose oxidase method with an automated glucose analyser. The remaining plasma was stored at $-20^{\circ} \mathrm{C}$ for later insulin dosage. Insulinaemia was 
determined by commercially available radioimmunoassays (Biodata S.p.A, Guidonia Montecelio, Roma, Italy).

\subsection{Model formulation}

The dynamic relation between plasma insulin and glucose concentration data detected during OGTTs were interpreted by means of a new model, which incorporates the classic minimal model of glucose kinetics [1] coupled with a gut compartment, which is supposed to account for the conversion of ingested glucose dose, $D(75 \mathrm{mg})$, into the rate of post hepatic glucose appearance into plasma $R_{a}(t)[11,12]$.

Our model is described by the following equations:

$$
\begin{array}{ll}
\frac{d G(t)}{d t}=-\left[S_{G}+X(t)\right] \cdot G(t)+S_{G} \cdot G_{b}+\frac{R_{a}(t)}{V W} ; & G(0)=G_{b} \\
\frac{d X(t)}{d t}=-p_{2} \cdot X(t)+p_{2} \cdot S_{I} \cdot\left[I(t)-I_{b}\right] ; & X(0)=0 \\
\frac{d G_{g}(t)}{d t}=R_{s}(t)-\left[R_{a}(t)+R_{l}(t)\right]=R_{s}(t)-k_{d} G_{g}(t) ; & G_{g}(0)=0
\end{array}
$$

where $G(t)$ is instantaneous glycaemia $(\mathrm{mg} / \mathrm{dl}) ; I(t)$ is instantaneous insulinaemia $(\mu \mathrm{U} / \mathrm{ml}) ; X(t)$ is insulin action proportional to insulinaemia deviation from basal in a remote compartment [1], $G_{b}$ and $I_{b}$ are baseline glycaemia and insulinaemia; $V W(\mathrm{dl})$ is glucose distribution volume; $S_{I}$ is insulin sensitivity $\left[\mathrm{min}^{-1} /\left(\mu \mathrm{U} \cdot \mathrm{ml}^{-1}\right)\right]$, $S_{G}$ is glucose effectiveness $\left(\mathrm{min}^{-1}\right)$ and $p_{2}$ is the rate constant $\left(\mathrm{min}^{-1}\right)$ which governs the speed of rise and decay of insulin action in the remote insulin compartment; $G_{g}(t)$ is glucose mass in the gut (mg); $R_{l}(t)$ accounts for the rate of splanchnic extraction $(\mathrm{mg} / \mathrm{min})$ of glucose and $R_{a}(t)$ is the rate of post hepatic appearance $(\mathrm{mg} / \mathrm{min})$ of ingested glucose into the systemic circulation. The sum of $R_{a}(t)$ and $R_{l}(t)$ is the rate of glucose delivery by the gut compartment, $R_{d}(t)$. The glucose flow which leaves the stomach and enters the gut compartment is denoted as $R_{S}(t)$ and is described by the following equation:

$$
R_{s}(t)=D \cdot \ln 2 \cdot 2^{-\left[\left(t+t_{i}\right) / \tau\right]^{\beta}} \cdot \frac{\beta}{\tau} \cdot\left(\frac{t+t_{i}}{\tau}\right)^{\beta-1} ; \quad 0 \leq \mathrm{t} \leq 300 \mathrm{~min}
$$

where the dimensionless parameter $\beta$ determines the shape of gastric retention decay curve, while $\tau(\mathrm{min})$ is the time lasting until $50 \%$ of the glucose dose, $D$, has been delivered. The $t_{i}$ parameter is assumed equal to 1 according to the observation that the administration of the dose $D$ started at minute -1 before the conventional start, $t=0^{+}$, of our experimental observation.

The rate of glucose delivery by the gut is assumed to be linearly related to the glucose mass in the gut via a delivery constant $k_{d}[11,12]$. Only a fraction of glucose delivered by the gut, however, enters the systemic circulation after passage through the liver. Based on dual tracer OGTT, a mean population value 
of 0.87 has been reported for $f$ [8]. Taking this value into account, the rate of appearance $(\mathrm{mg} / \mathrm{min})$ of ingested glucose into plasma can be expressed as:

$$
R_{a}(t)=k_{a b s} \cdot G_{g}(t)
$$

where the parameter

$$
k_{a b s}=f \cdot k_{d}
$$

portrays the physiological meaning of the rate constant of systemic glucose absorption from the gut.

\subsection{Parameter estimation}

Our model is characterised by eight free parameters: $\beta, \tau, k_{d}, f, S_{G}, S_{I}, p_{2}$ and $V W$. The product of $f$ and $k_{d}$ yields $k_{a b s}$ (eq. (6)). Suitable estimates of $\beta$ and $\tau$ were determined by fitting equation (4) to the mean values of the rate of glucose delivery into the duodenum reported by Schirra et al. [13]. For $D=75 \mathrm{~g}$, we found $\beta=1.15$ and $\tau=43 \mathrm{~min}$. These values allowed simulation of the $R_{S}(t)$ profile (Fig. 1) to be filled into equation (3).

From a priori identifiability analysis [14] we found that unique identifiability of parameters $S_{I}, p_{2}$ and $k_{d}$ can be accomplished if $f, S_{G}$ and $V W$ are also known.

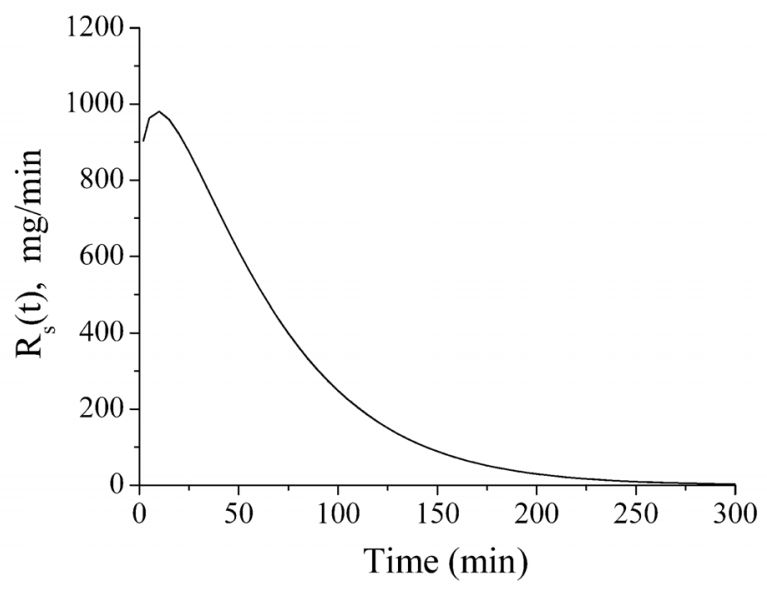

Figure 1: Time course of the curve of glucose delivery into the duodenum, after oral ingestion of a glucose dose $D=75 \mathrm{~g}$, as described by equation (4) with $\beta=1.15$ and $\tau=43 \mathrm{~min}$.

In accordance with estimates reported by Dalla Man [8] from model independent, dual tracer OGTT studies, we assumed reference population values 
of $f=0.87, S_{G}=0.028 \mathrm{~min}^{-1}$ and $V=1.34 \mathrm{dl} / \mathrm{kg}$. The product of $V$ times body weight, $B W(\mathrm{~kg})$, of individual subjects who underwent the OGTT for the present study yielded $V W$ values (dl) to be filled into equation (1). Estimation of $S_{I}, k_{d}$ and $p_{2}$ was accomplished making use of a weighted non-linear estimation technique implemented by the SAAM II software (SAAM Institute, University of Washington, Seattle, WA) [15]. Weights were optimally chosen, i.e., equal to the inverse of the variance of the glucose measurement errors. The errors associated with total glucose measurements were assumed to be normally distributed random variables with zero mean and a constant percent coefficient of variation (CV\%) equal to $2 \%$. Maximum a posteriori Bayesian estimation was employed for $p_{2}$ assumed normally distributed with mean $0.012 \mathrm{~min}^{-1}$ and $\mathrm{CV} \%=20 \%[7,8]$. To assess the goodness of glucose data fit we analyzed the weighted residuals, i.e. the differences between the data and model-predicted values, multiplied by the square root of a weight proportional to the reciprocal of datum SD.

\subsection{Reproducibility analysis}

Intraclass correlation coefficient [16] was used to evaluate the agreement of paired sample sets $\left(\mathrm{OGTT}_{300 / 22}\right.$ and $\left.\mathrm{OGTT}_{300 / 11}\right)$ in providing $S_{I}$ and $k_{a b s}$ estimates, irrespective of CV\%, after application of our model. Means and $95 \%$ confidence intervals (CI) of estimated indexes were also reported.

\section{Results}

On average, fasting glycaemia and insulinaemia were $80.2(\mathrm{SD}=9.0) \mathrm{mg} / \mathrm{dl}$ and $5.58(\mathrm{SD}=2.60) \mu \mathrm{U} / \mathrm{ml}$, respectively. Triglycerides and HDL cholesterol levels were $88.0(\mathrm{SD}=52.5) \mathrm{mg} / \mathrm{dl}$ and $57.0(\mathrm{SD}=12.6) \mathrm{mg} / \mathrm{dl}$, respectively.

Data fit was good as judged from the mean weighted residuals which showed no systematic deviation from zero and were within the range $[-1,+1]$ as displayed in Fig. 2.

Mean estimates (CI) of $S_{I}^{22}, k_{d}^{22}$ and $p_{2}^{22}$ obtained from the full OGTT $\mathrm{O}_{300 / 22}$ data set were $12.3(8.8-15.8) \times 10^{-4} \mathrm{~min}^{-1} /\left(\mu \mathrm{U} \cdot \mathrm{ml}^{-1}\right), 2.07(1.51-2.63) \times 10^{-2} \mathrm{~min}^{-1}$ and $1.08(0.86-1.30) \times 10^{-2} \mathrm{~min}^{-1}$, respectively. Mean CV\% values were $12.1 \%$ $(\mathrm{SD}=2.9)$ for $S_{I}^{22}, 17.2 \%(\mathrm{SD}=2.4)$ for $k_{d}^{22}$ and $20.9 \%(\mathrm{SD}=4.1)$ for $p_{2}^{22}$. Mean estimates $(\mathrm{CI})$ of $S_{I}^{11}, k_{d}^{11}$ and $p_{2}^{11}$ obtained from the reduced 11-sample data subset were $11.3(7.6-15.0) \times 10^{-4} \mathrm{~min}^{-1} /\left(\mu \mathrm{U} \cdot \mathrm{ml}^{-1}\right), 2.01(1.36-2.66) \times 10^{-2} \mathrm{~min}^{-1}$ and $1.16(0.99-1.33) \times 10^{-2} \mathrm{~min}^{-1}$, respectively. Mean $\mathrm{CV} \%$ values were $12.6 \%$ $(\mathrm{SD}=5.1)$ for $S_{I}^{11}, 20.4 \%(\mathrm{SD}=4.6)$ for $k_{d}^{11}$ and $23.5 \%(\mathrm{SD}=3.5)$ for $p_{2}^{11}$. Mean (CI) values of $k_{a b s}^{22}$ and $k_{a b s}^{11}$, computed by equation (6), were $1.80(1.31-2.29)$ $\times 10^{-2} \mathrm{~min}^{-1}$ and $1.75(1.19-2.31) \times 10^{-2} \mathrm{~min}^{-1}$, respectively. 
Intraclass correlation coefficient was 0.93 for $S_{I}^{11}$ compared with $S_{I}^{22}$ values, and 0.97 for $k_{a b s}^{11}$ compared with $k_{a b s}^{22}$.

After determination of all model parameters we took advantage of our modelling of glucose delivery by the gut to predict (eq. (5)) the time course of the rate of glucose appearance into plasma, $R_{a}(t)$ in individuals. Because $G_{g}(t)$ is expressed in $\mathrm{mg}$ and $k_{a b s}$ in $\min ^{-1}, R_{a}(t)$ was normalised for body weight to obtain a profile in $\mathrm{mg} \cdot \mathrm{min}^{-1} / \mathrm{kg}$ comparable with data in the literature (see Discussion). The mean profiles provided by the model using the parameters obtained from considering the OGTT $_{300 / 22}$ (continuous line) and the OGTT $_{300 / 11}$ (dashed line) data sets are shown in Fig. 3. The two curves are practically superimposed and show a bell shape with a steep rise to a peak, occurring about the $60^{\text {th }} \mathrm{min}$, followed by a slow decrease to zero in about four hours.

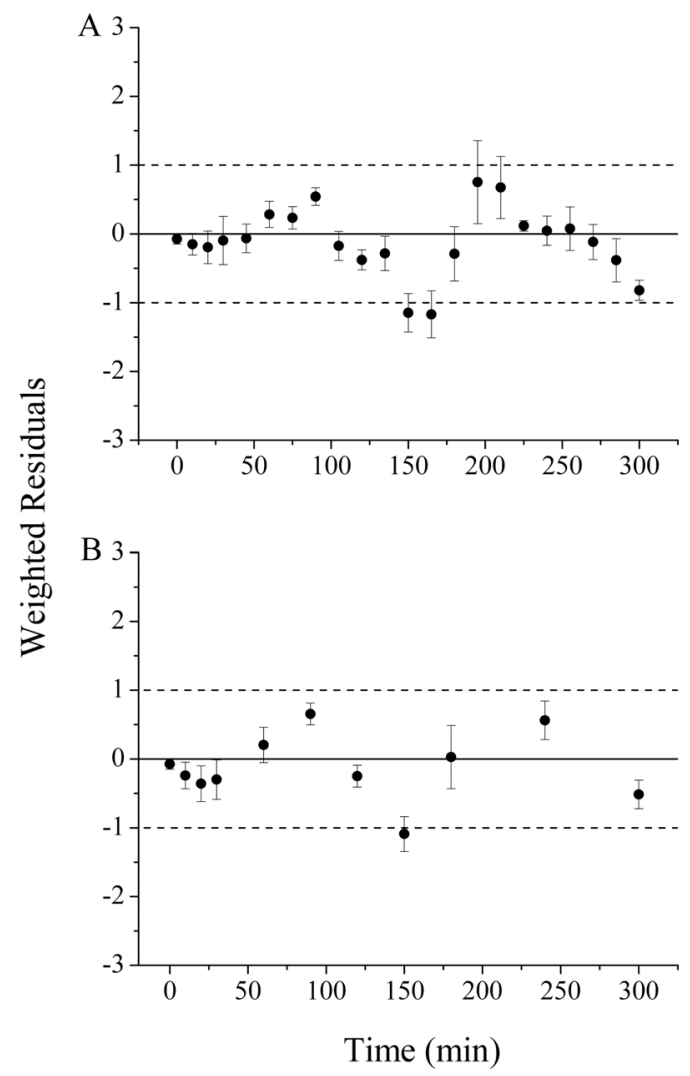

Figure 2: Average ( $\pm \mathrm{SE}$ ) weighted residuals (closed circles) as produced by fitting our model to the full 22-sample data set (A: OGTT $\left._{300 / 22}\right)$ and to the reduced 11-sample data subset (B: OGTT 300/11 . 


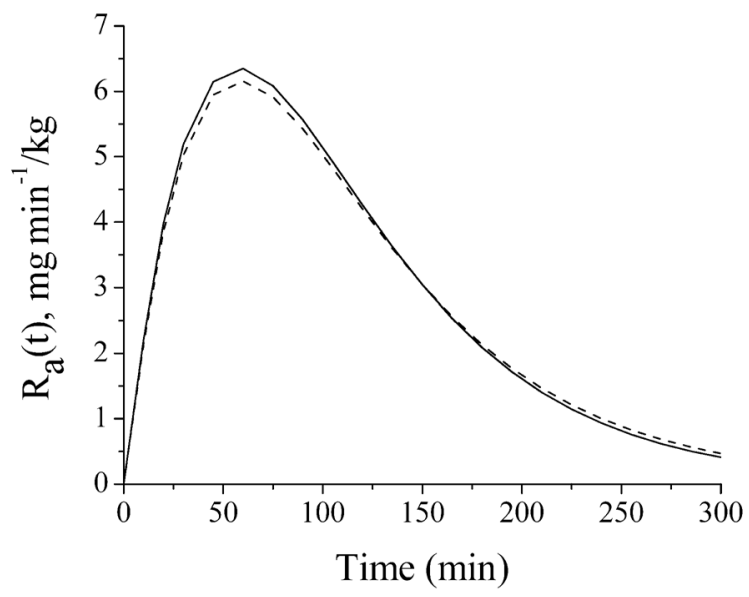

Figure 3: Comparison between average rate of glucose appearance into plasma predicted by our OGTT minimal model after fitting to the full 22-sample data set (continuous line) and after fitting to the reduced 11-sample data subset (dashed line).

\section{Discussion}

The process of setting up a model that allows estimation of insulin sensitivity from oral glucose tests enhances the problem of the description of the rate of appearance of glucose into plasma, $R_{a}(t)$, [5-9]. To resolve this problem we coupled the minimal model of glucose kinetics, developed for FSIGTT-based studies with a monocompartmental model of the gut that describes the conversion into $R_{a}(t)$ of a dose, $D$, of glucose ingested during an OGTT. A new mathematical representation (eq. (4)) was introduced to describe the glucose delivery to the gut. This yielded a significant improvement over the function previously assumed by us [9] in fitting the data reported by Schirra et al. [13]. A further improvement was achieved by taking into account that only a fraction, $f=$ 0.87 [8], of glucose delivered by the gut enters the systemic circulation after passage through the liver, such that the rate of appearance of glucose into plasma is characterized by the rate constant $k_{a b s}$ which is $87 \%$ of the rate constant, $k_{d}$, of glucose delivery by the gut. Our model allows estimation of these constants, thus providing information of physiological and clinical interest on glucose delivery to the systemic circulation besides information on the sum effects of insulin to enhance glucose uptake by peripheral tissues and, concomitantly, to suppress liver glucose output.

The ability of our model to provide suitable estimates of these metabolic parameters was tested in subjects who had no history of diabetes mellitus. In selecting the participants to this study we made reference to the ATP III criteria [10] to avoid the confounding effects of MS as a state associated with excess risk of diabetes and cardiovascular disease. 
Estimates of insulin sensitivity provided by our model from fitting to the full 22-sample data set $\left(\mathrm{OGTT}_{300 / 22}\right)$ can be compared with estimates reported by others after application to normal subjects of other model based methods such as the "integral equation" [5] and the "differential equation" methods [7,8]. Because the "integral equation" method produces an insulin sensitivity index which has the same units, $\mathrm{dl} \cdot \mathrm{kg}^{-1} \cdot \mathrm{min}^{-1} /(\mu \mathrm{U} / \mathrm{ml})$, of the analogous clamp index, a suitable comparison requires that the $S_{I}$ values produced by our present method and the differential equation method are multiplied by $V(\mathrm{dl} / \mathrm{kg})$. The mean (CI) value of $S_{I}^{22} \cdot V, 16.5(11.8-21.2) \times 10^{-4} \mathrm{dl} \cdot \mathrm{kg}^{-1} \cdot \mathrm{min}^{-1} /(\mu \mathrm{U} / \mathrm{ml})$, produced by our model compares well with the mean value of $16.1(13.9-18.2) \times 10^{-4} \mathrm{dl} \cdot \mathrm{kg}^{-1} \cdot \mathrm{min}^{-}$ $1 /(\mu \mathrm{U} / \mathrm{ml})$ reported by Dalla Man [8] for 88 NGT subjects, and the mean value of $13.6(5.9-21.3) \times 10^{-4} \mathrm{dl} \cdot \mathrm{kg}^{-1} \cdot \mathrm{min}^{-1} /(\mu \mathrm{U} / \mathrm{ml})$ reported by Caumo et al [5] for 10 normal subjects. This finding is promising in view of a more thoroughly comparison of our and these previous methods by direct application to our data set.

Easier and less costly applications of oral glucose tests, interpreted with oral minimal models, can be accomplished by reduction of the sampling schedule from 22 to 11 . The intraclass correlation coefficients demonstrated a substantial agreement of the two paired methods for estimation of $S_{I}$ and $k_{a b s}$ parameters. Further reproducibility analysis involving the $\mathrm{CV} \%$ associated with individual parameter estimates is desirable to assess the precision of estimated intraclass correlation coefficients and complete the analysis of agreement.

In conceptual terms our new model remains essentially a "differential method" as that first proposed by Dalla Man et al. [7, 8]. A significant difference is that, in our approach, a profile, $R_{s}(t)$, of glucose delivery into the duodenum is assumed (eq. (4)), whereas the "differential method" assumes a $R_{a}(t)$ profile.

An advantage of our approach is that the rate of appearance of glucose into plasma is governed by a physiologically meaningful parameter, $k_{a b s}$. Our mean (CI) estimated values of $k_{a b s}^{22}, 1.80(1.31-2.29) \times 10^{-2} \mathrm{~min}^{-1}$, and $k_{a b s}^{11}, 1.75$ (1.192.31) $\times 10^{-2} \mathrm{~min}^{-1}$, compare well with the value of $1.66 \times 10^{-2} \mathrm{~min}^{-1}$ calculated by Lehmann and Deutsch [11] from Berger and Rodbard [17] and Guyton et al. [18], and used for the AIDA simulation software.

Our estimates of $k_{a b s}$ allow prediction (eq. (5)) of the profile of the rate of glucose appearance into plasma, $R_{a}(t)$. On average, the profile obtained after application of our model to the $\mathrm{OGTT}_{300 / 22}$ data set is practically superimposed to that obtained from the $\mathrm{OGTT}_{300 / 11}$ data set (Fig.3). Qualitative comparison of this profile with that reported by others from dual tracer OGTT study and with its prediction by the oral minimal model incorporating a piecewise approximation of $R_{a}(t)$ [7,8] shows a discrepancy in the first thirty minutes. The sharp initial peak which characterizes these reported profiles cannot be reproduced by our oral minimal model. More thoroughly comparison is needed to explain the cause of this discrepancy.

In conclusion, our new model for oral glucose tests appears a potentially useful tool to measure insulin sensitivity and the rate of appearance of glucose into plasma as it relates to glucose delivery by the gut. The present application of 
our model to normal humans shows that our approach is applicable to a reduced protocol with 11 samples in 5 hours. Further studies are needed to evaluate the sensitivity of model output to alterations of fixed parameters and to asses the applicability of our model in disease states. Integration with information which can be obtained from the alternative "differential equation" approach $[7,8]$ can contribute to a decisive improvement in the description and interpretation of glucose-insulin system dynamics in health and disease.

\section{Acknowledgements}

This work was supported in part by the Italian Ministry of Instruction, University and Research (PRIN-COFIN 2004 grant to R. Burattini).

\section{References}

[1] Bergman, R. N., Ider, Y. Z., Bowden, C. R. \& Cobelli, C., Quantitative estimation of insulin sensitivity. Am. J. Physiol., 236, pp. E667-E677, 1979.

[2] Saad, M. F., Anderson, R. L., Laws, A., Watanabe, R. M., Kades, W. W., Chen, Y.-D. I., Sands, R. E., Pei, D., Savage, P. J. \& Bergman, R. N., A comparison between the minimal model and the glucose clamp in the assessment of insulin sensitivity across the spectrum of glucose tolerance. Diabetes, 43(9), pp. 1114-1121, 1994.

[3] Finegood, D. T., Application of the minimal model of glucose kinetics. The Minimal Model Approach and Determinants of Glucose Tolerance, ed. Bergman, R. N. \& Lovejoy, J. C.: Louisiana State Univ. Press, Baton Rouge, LA, pp. 51-122, 1997.

[4] Natalucci, S., Boemi,. M, Fumelli, P., Testa, I., Fumelli, D. \& Burattini R., One- and two-compartment minimal models detect similar alterations of glucose metabolism indexes in hypertension. Metabolism, 49, pp. 15291536, 2000.

[5] Caumo, A., Bergman, R. N. \& Cobelli, C., Insulin sensitivity from meal tolerance test in normal subjects: a minimal model index. J. Clin. Endocrinol. Metab., 85(11), pp. 4396-4402, 2000.

[6] Breda, E., Kavaghan, M. K., Toffolo, G., Polonsky K. S. \& Cobelli, C., Oral glucose tolerance test minimal model indexes of beta-cell function and insulin sensitivity. Diabetes, 50, pp. 150-158, 2001.

[7] Dalla Man, C., Caumo A. \& Cobelli, C., The oral glucose minimal model: estimation of insulin sensitivity from a meal test. IEEE Trans. Biomed. Eng., 49(5), pp. 419-429, 2002.

[8] Dalla Man, C., Oral Glucose minimal models: formulation, validation and use, Ph.D. Thesis, University of Padua, Italy, 2004.

[9] Natalucci, S., Di Nardo, F., Staffolani, P., De Marzi, C., Morosini, P. \& Burattini, R., Glucose absorption and insulin sensitivity from oral glucose tolerance test. Proc. of the IEEE-EMB $25^{\text {th }}$ Annual Conference, pp. 2758$2760,2003$. 
[10] Expert Panel on Detection, Evaluation, and Treatment of High Blood Cholesterol in Adults: Executive Summary of the Third Report of the National Cholesterol Education Program (NCEP) Expert Panel on Detection, Evaluation, and Treatment of High Blood Cholesterol in Adults (Adult Treatment Panel III). JAMA, 285, pp. 2486-97, 2001.

[11] Lehmann E. D. \& Deutsch, T., A physiological model of glucose-insulin interaction in type 1 diabetes mellitus. J. Biomed. Eng., 14, pp. 235-242, 1992.

[12] Worthington D. R., Minimal model of food absorption in the gut. Med. Inform., 22(1), pp. 35-45, 1997.

[13] Schirra, J., Katschinski, M., Weidmann, C., Schäfer, T., Wank, U., Arnold R. \& Göke, B., Gastric emptying and release of incretin hormones after glucose ingestion in humans. J. Clin. Invest., 87(1), pp. 92-103, 1996.

[14] Carson, E. R., Cobelli, C., \& Finkelstein, L., The Mathematical Modeling of Metabolic and Endocrine Systems, Wiley: New York, 1983.

[15] Barrett, P. H., Bell, B. M., Cobelli, C., Golde, H., Schumitzky, A., Vicini P. \&. Foster, D.M., SAAM II: simulation, analysis, and modelling software for tracer and pharmacokinetics studies. Metabolism, 47(4), pp. 484-492, 1998.

[16] Fleiss J. L., Statistical Methods for Rates and Proportion, Wiley: New York, pp. 212-236, 1980.

[17] Berger, M. \& Rodbard, D., Computer simulation of plasma insulin and glucose dynamics after subcutaneous insulin injection. Diabetes Care, 12, pp. 725-736, 1989.

[18] Guyton, J. R., Foster, R. O., Soeldner, J. S., Tan, M. H., Kahn, C. B., Koncz L. \& Gleason, R. E., A model of glucose-insulin homeostasis in man that incorporates the heterogeneous fast pool theory of pancreatic insulin release. Diabetes, 27, pp. 1027-1042, 1978. 